\title{
A fixed point result and the stability problem in Lie superalgebras
}

\author{
Madjid Eshaghi ${ }^{1,2}$, Sadegh Abbaszadeh ${ }^{1,3^{*}}$, Manuel de la Sen ${ }^{4}$ and Zahra Farhad
}

\author{
"Correspondence: \\ abbaszadeh@semnan.ac.ir \\ ${ }^{1}$ Department of Mathematics, \\ Faculty of Mathematics, Statistics \\ and Computer Sciences, Semnan \\ University, Semnan, 35195-363, Iran \\ ${ }^{3}$ Young Researchers and Elite Club, \\ Malayer Branch, Azad University, \\ Malayer, Iran \\ Full list of author information is \\ available at the end of the article
}

\begin{abstract}
In this paper, we introduce the concept of normed Lie superalgebras and define the superhomomorphism and the superderivation in normed Lie superalgebras. We define a generalized $T$-orbitally complete metric space and prove a new fixed point alternative concerning the stability problem of functional equations. Consequently, we deal with the stability of the superhomomorphism and the superderivation in Lie superalgebras using that new fixed point result. In addition, we find some conditions under which an approximate superhomomorphism or superderivation is an exact superhomomorphism or superderivation.
\end{abstract}

MSC: Primary 47H10; 39B82; secondary 16W25

Keywords: Lie superalgebra; superhomomorphism; superderivation; T-orbitally complete metric space

\section{Introduction}

Most physicists believe that all of the familiar particle interactions, weak and electromagnetic as well as strong interactions, are associated with a Lie algebra in similar ways. This suggests that it may be possible to unify all the particle interactions as different aspects of a single underlying interaction, based on a single simple Lie algebra. With an increasing amount of theory and applications concerning Lie algebras of various dimensions, it is becoming necessary to ascertain which tools are applicable for handling them. The miscellaneous characteristics of Lie algebras constitute such tools and have also found applications: Casimir operators [1], derived, lower central and upper central sequences, the Lie algebra of derivations, radicals, nilradicals, ideals, subalgebras [2, 3], and recently megaideals [4]. These characteristics are particularly crucial when considering possible connections among Lie algebras. Physically motivated relations between two Lie algebras, namely contractions and deformations, have been extensively studied; see e.g. [5-7].

The concept of Lie algebra can be expressed in several different ways. The most familiar are in terms of generators and relations and in terms of a bilinear bracket on a vector space $V$ satisfying the Jacobi identity. In physical notation, let $X_{a}$ be a basis for $V$. The bracket $[\cdot, \cdot]$ can be specified by structure constants $C_{a b}^{c}$ via the formula

$$
\left[X_{a}, X_{b}\right]=C_{a b}^{c} X_{c}
$$

The structure constants are skew-symmetric in the lower indices $a, b$.

(c) 2015 Eshaghi et al. This article is distributed under the terms of the Creative Commons Attribution 4.0 International License (http://creativecommons.org/licenses/by/4.0/), which permits unrestricted use, distribution, and reproduction in any medium, provided you give appropriate credit to the original author(s) and the source, provide a link to the Creative Commons license, and indicate if changes were made. 
The derivation of a new Lie superalgebra from given Lie algebras is particularly interesting in physics, since it allows us to find new physical theories from already known theories. The presentation of Lie superalgebras by a finite set of generators and defining relations is one of the most general mathematical and algorithmic schemes of their analysis. It is very important, for instance, for the investigation of the particular Lie superalgebras arising in different supersymmetric physical models. Palev [8] showed that the position and momentum operators in quantum mechanics and fields in quantum field theory are the generators of finite-dimensional and infinite-dimensional orthosymplectic Lie superalgebras, respectively.

In mathematical analysis, the stability problem is presented as follows: Assume that a mathematical object satisfies a certain property approximately according to some convention. Is it then possible to find near this object an object satisfying the property accurately? The interested reader can find more information on such problems with the emphasis on functional equations in [9-11].

In the theory of functional equations, there are cases in which each approximate function is actually a true function. In such cases, we say that the functional equation is hyperstable. Indeed, a functional equation is hyperstable if every solution satisfying the equation approximately is an exact solution of it. For the history and various aspects of this theory we refer the reader to [12-16].

In this paper, we introduce the concept of normed Lie superalgebras. For a continuous self-mapping $T$, we define a generalized $T$-orbitally complete metric space and prove a new fixed point result. We define a superhomomorphism and a superderivation and investigate the stability of these linear mathematical objects in Lie superalgebras using the new fixed point alternative. Moreover, we find some conditions under which an approximately object is an exact object.

The paper is organized as follows: The concept of normed Lie superalgebras is introduced in Section 2. In Section 3, generalized $T$-orbitally complete metric spaces are defined and a new alternative of fixed point is presented. In Section 4, we begin to discuss the stability of the superhomomorphism in Lie superalgebras. Moreover, we find conditions under which an approximate superhomomorphism is an exact superhomomorphism. In Section 5, we deal with the stability of the superderivation in Lie superalgebras. Moreover, we find conditions under which an approximate superderivation is an exact superderivation. In Section 6, an application of the new fixed point theorem to prove the stability of functional equations is stated.

\section{Lie superalgebras}

Let $\mathbb{Z}_{2}=\{\overline{0}, \overline{1}\}$ denote the group of two elements. A superalgebra $\mathcal{G}=\mathcal{G}_{\overline{0}} \oplus \mathcal{G}_{\overline{1}}$, sometimes also called a $\mathbb{Z}_{2}$-graded algebra, is a direct sum of two spaces $\mathcal{G}_{\overline{0}}$ and $\mathcal{G}_{\overline{1}}$ equipped with a bilinear multiplication satisfying $\mathcal{G}_{i} \mathcal{G}_{j} \subseteq \mathcal{G}_{i+j}$, for $i, j \in \mathbb{Z}_{2}$. Elements $x \in \mathcal{G}_{\overline{0}}$ are called even or of degree $|x|=0$ while elements $x \in \mathcal{G}_{\overline{1}}$ are called odd or of degree $|x|=1$. The interested reader can find more information about superalgebras in [17-20].

A Lie superalgebra is an algebra $\mathcal{L}=\mathcal{L}_{\overline{0}} \oplus \mathcal{L}_{\overline{1}}$ with bilinear multiplication $\mu: \mathcal{L}^{2} \rightarrow \mathcal{L}$ satisfying the following two axioms: for $a, b, c \in \mathcal{L}_{\overline{0}} \cup \mathcal{L}_{\overline{1}}$ :

(1) Skew-supersymmetry: $\mu(a, b)=-(-1)^{|a| \cdot|b|} \mu(b, a)$.

(2) Super Jacobi identity: $\mu(a, \mu(b, c))=\mu(\mu(a, b), c)+(-1)^{|a| \cdot|b|} \mu(b, \mu(a, c))$. 
Definition 2.1 Let $\mathcal{L}=\mathcal{L}_{\overline{0}} \oplus \mathcal{L}_{\overline{1}}$ and $\mathcal{L}^{\prime}=\mathcal{L}_{\overline{0}}^{\prime} \oplus \mathcal{L}_{\overline{1}}^{\prime}$ be Lie superalgebras with multiplications $\mu$ and $v$, respectively. Additive functions $H_{i}: \mathcal{L} \rightarrow \mathcal{L}^{\prime}$ are called homomorphisms of degree $i$ if

$$
H_{i}(\mu(x, y))=v\left(H_{i}(x), H_{i}(y)\right)
$$

for all $x, y \in \mathcal{L}_{\overline{0}} \cup \mathcal{L}_{\overline{1}}$. A superhomomorphism $H: \mathcal{L} \rightarrow \mathcal{L}^{\prime}$ by $H=H_{\overline{0}}+H_{\overline{1}}$ is the sum of a homomorphism of degree 0 and a homomorphism of degree 1 .

Definition 2.2 Let $\mathcal{L}=\mathcal{L}_{\overline{0}} \oplus \mathcal{L}_{\overline{1}}$ be a Lie superalgebra with multiplication $\mu$. Additive functions $D_{i}: \mathcal{L} \rightarrow \mathcal{L}$ are called derivation of degree $i$ if

$$
D_{i}(\mu(x, y))=\mu\left(D_{i}(x), y\right)+(-1)^{i|x|} \mu\left(x, D_{i}(y)\right)
$$

for all $x, y \in \mathcal{L}_{\overline{0}} \cup \mathcal{L}_{\overline{1}}$. A superderivation $D: \mathcal{L} \rightarrow \mathcal{L}$ by $D=D_{0}+D_{1}$ is the sum of a derivation of degree 0 and a derivation of degree 1 .

Definition 2.3 Let $\mathcal{L}$ be a Lie superalgebra with multiplication $\mu$. The commutant of $\mathcal{L}$ is the subset $\mathcal{L}^{c}$ of $\mathcal{L}$ given by

$$
\mathcal{L}^{c}=\{a \in \mathcal{L}: \mu(x, a)=\mu(a, x), x \in \mathcal{L}\} .
$$

\section{A fixed point result}

Let $(X, d)$ be a metric space. The celebrated Banach contraction theorem (Banach [21], 1922) ensures us of a unique fixed point if a mapping $T: X \rightarrow X$ is a contraction, i.e., if there exists a positive number $q<1$ such that

$$
d(T x, T y) \leq q d(x, y)
$$

for all $x, y \in X$. The Banach contraction theorem formulated for a complete metric space is one of the most simple and, at the same time, the most important method for the existence and uniqueness of solution of nonlinear problems arising in mathematics and its applications to engineering and natural sciences [22-25].

In 1991, Baker [12] used the Banach fixed point theorem to prove the Hyers-Ulam stability. The method was generalized by Radu [26] and since then has been used by many authors (see [27-29]). We prove a new theorem about this fundamental result as follows.

Let $X$ be a nonempty set and $d: X \times X \rightarrow[0, \infty]$ be a function satisfying the following conditions:

(1) $d(x, y)=0$ if and only if $x=y$;

(2) $d(x, y)=d(y, x)$ (symmetry);

(3) $d(x, y) \leq d(x, z)+d(z, y)$ (triangular inequality),

for all $x, y, z \in X$. Then $(X, d)$ is called a generalized metric space.

Definition 3.1 (Ćirić [30-32]) Let $T$ be a self-mapping of a generalized metric space $(X, d)$. If for any $x \in X$, every Cauchy sequence of the orbit

$$
O_{x}(T)=\left\{x, T x, T^{2} x, \ldots\right\}
$$

is convergent in $X$, then $(X, d)$ is called a generalized $T$-orbitally complete metric space. 
Definition 3.2 (Ćirić [30-32]) Let $T$ be a self-mapping of a metric space $(X, d)$. We say $T$ is orbitally continuous if and only if $\lim _{n \rightarrow \infty} T^{n} x=u \in X$ implies $T u=\lim _{n \rightarrow \infty} T^{n} x$.

Theorem 3.3 (Fixed point theorem) Suppose that $(X, d)$ is a generalized T-orbitally complete metric space and $T$ is an orbitally continuous self-mapping such that

$$
d\left(T x, T^{2} x\right) \leq q d(x, T x)
$$

for any $x \in X$ and fixed constant $q$ with $0<q<1$. Then the following alternative holds: either

(a) for all $n \geq 0, d\left(T^{n} x, T^{n+1} x\right)=\infty$, or

(b) there exists a positive integer $N$ such that $d\left(T^{n} x, T^{n+1} x\right)<\infty$ for all $n \geq N$.

In this case, the sequence $\left(T^{n} x\right)_{n \in \mathbb{N}}$ is convergent to a fixed point $x^{*}$ of T. Moreover, if $d(x, T x)<\infty$, then

$$
d\left(x, x^{*}\right) \leq \frac{1}{1-q} d(x, T x)
$$

Proof For any $x \in X$, consider the $T$-orbit $\left\{x, T x, T^{2} x, \ldots\right\}$. Then either for all $n \geq 0$, $d\left(T^{n} x, T^{n+1} x\right)=\infty$, or there exists a positive integer $N$ such that

$$
d\left(T^{n} x, T^{n+1} x\right)<\infty
$$

for all $n \geq N$. In order to show that $\left(T^{n} x\right)_{n \in \mathbb{N}}$ is convergent in $X$, we need to show that $\left(T^{n} x\right)_{n \in \mathbb{N}}$ is Cauchy in $X$. Using the inequality (3), for all $n \geq N$ we have

$$
d\left(T^{n} x, T^{n+1} x\right) \leq q d\left(T^{n-1} x, T^{n} x\right) \leq \cdots \leq q^{n-N} d\left(T^{N} x, T^{N+1} x\right)
$$

Thus, for all $n, m \in \mathbb{N}$ with $n>m \geq N$, we get

$$
\begin{aligned}
d\left(T^{m} x, T^{n} x\right) & \leq d\left(T^{m} x, T^{m+1} x\right)+\cdots+d\left(T^{n-1} x, T^{n} x\right) \\
& \leq q^{m-N} d\left(T^{N} x, T^{N+1} x\right)+\cdots+q^{n-N-1} d\left(T^{N} x, T^{N+1} x\right) \\
& =\left(q^{m-N}+\cdots+q^{n-N-1}\right) d\left(T^{N} x, T^{N+1} x\right) .
\end{aligned}
$$

As $m, n$ tend to infinity, we conclude that $\left(T^{n} x\right)_{n \in \mathbb{N}}$ is Cauchy. Since $X$ is $T$-orbitally complete metric space, then $\left(T^{n} x\right)_{n \in \mathbb{N}}$ converges to some $x^{*} \in X$. We show that $x^{*}$ is a fixed point of $T$. Indeed, by Definition 3.2 we have

$$
T\left(x^{*}\right)=\lim _{n \rightarrow \infty} T^{n+1}(x)=x^{*}
$$

It remains to show that the inequality (4) is sharp. Indeed, for all $n \in \mathbb{N}$ and fixed constant $q$ with $0<q<1$,

$$
d\left(x, T^{n} x\right) \leq d(x, T x)+\cdots+d\left(T^{n-1} x, T^{n} x\right) \leq\left[1+\cdots+q^{n-1}\right] d(x, T x) .
$$

Taking the limit as $n \rightarrow \infty$, we obtain (4). 
As a result of Theorem 3.3, we prove the Hyers-Ulam stability of the superhomomorphism and the superderivation in Lie superalgebras, using the above mentioned fixed point alternative. For more details as regards the stability problems in Lie algebras, we refer the reader to $[33,34]$.

\section{The superhomomorphism in Lie superalgebras}

Let $\varphi: \mathcal{L}^{2} \rightarrow(0, \infty)$ and $\psi: \mathcal{L}^{2} \rightarrow[0, \infty)$ be functions for which there exists $0<q=q(\ell)<$ 1 such that

$$
\begin{aligned}
& \varphi\left(2^{\ell} x, 2^{\ell} y\right) \leq 2^{\ell} q \varphi(x, y), \\
& \psi\left(2^{\ell} x, y\right) \leq 2^{\ell} q \psi(x, y), \quad \psi\left(x, 2^{\ell} y\right) \leq 2^{\ell} q \psi(x, y)
\end{aligned}
$$

for all $x, y \in \mathcal{L}_{\overline{0}} \cup \mathcal{L}_{\overline{1}}$ and $\ell \in\{-1,1\}$.

Theorem 4.1 Let $\mathcal{L}=\mathcal{L}_{\overline{0}} \oplus \mathcal{L}_{\overline{1}}$ be a Lie superalgebra with multiplication $\mu$ and norm $\|\cdot\|_{\mathcal{L}}$ and $\mathcal{L}^{\prime}=\mathcal{L}_{\overline{0}}^{\prime} \oplus \mathcal{L}_{\overline{1}}^{\prime}$ be a complete Lie superalgebra with multiplication $v$ and norm $\|\cdot\|_{\mathcal{L}^{\prime}}$. Suppose that $f_{i}: \mathcal{L} \rightarrow \mathcal{L}^{\prime}\left(i \in \mathbb{Z}_{2}\right)$ are functions satisfying

$$
\begin{aligned}
& \left\|f_{i}(x+y)-f_{i}(x)-f_{i}(y)\right\|_{\mathcal{L}^{\prime}} \leq \varphi(x, y), \\
& \left\|f_{i}(\mu(x, y))-v\left(f_{i}(x), f_{i}(y)\right)\right\|_{\mathcal{L}^{\prime}} \leq \psi(x, y)
\end{aligned}
$$

for all $x, y \in \mathcal{L}_{\overline{0}} \cup \mathcal{L}_{\overline{1}}$, where $\varphi: \mathcal{L}^{2} \rightarrow(0, \infty)$ and $\psi: \mathcal{L}^{2} \rightarrow[0, \infty)$ are functions satisfying the contractive conditions (5) and (6), for $0<q=q(\ell)<1$. Then there exists a unique superhomomorphism $H^{\ell}: \mathcal{L} \rightarrow \mathcal{L}^{\prime}$ with $H^{\ell}=H_{\overline{0}}^{\ell}+H_{\overline{1}}^{\ell}$ such that

$$
\left\|H_{i}^{\ell}(x)-f_{i}(x)\right\|_{\mathcal{L}^{\prime}} \leq \frac{q^{\frac{1-\ell}{2}}}{2(1-q)} \varphi(x, x)
$$

for all $x \in \mathcal{L}_{\overline{0}} \cup \mathcal{L}_{\overline{1}}, \ell \in\{-1,1\}$, and $i \in \mathbb{Z}_{2}$.

Proof We define $\mathcal{E}:=\left\{g: \mathcal{L} \rightarrow \mathcal{L}^{\prime}: g(0)=0\right\}$ and a generalized metric on $\mathcal{E}:$

$$
d(g, h)=\sup _{x \in \mathcal{L}_{\overline{0}} \cup \mathcal{L}_{\overline{1}}} \frac{\|g(x)-h(x)\|_{\mathcal{L}^{\prime}}}{\varphi(x, x)} .
$$

Consider the linear mapping $T: \mathcal{E} \rightarrow \mathcal{E}$ defined as

$$
T_{\ell} g(x)=2^{-\ell} g\left(2^{\ell} x\right)
$$

for all $x \in \mathcal{L}_{\overline{0}} \cup \mathcal{L}_{\overline{1}}$ and $\ell \in\{-1,1\}$. We can write, for any $f \in \mathcal{E}$,

$$
\begin{aligned}
\frac{\left\|T_{\ell} f(x)-T_{\ell}^{2} f(x)\right\|_{\mathcal{L}^{\prime}}}{\varphi(x, x)} & =\frac{\left\|2^{-\ell} f\left(2^{\ell} x\right)-2^{-2 \ell} f\left(2^{2 \ell} x\right)\right\|_{\mathcal{L}^{\prime}}}{\varphi(x, x)} \\
& =\frac{2^{-\ell}\left\|f\left(2^{\ell} x\right)-2^{-\ell} f\left(2^{2 \ell} x\right)\right\|_{\mathcal{L}^{\prime}}}{\varphi(x, x)} \\
& \leq q \frac{\left\|f\left(2^{\ell} x\right)-2^{-\ell} f\left(2^{2 \ell} x\right)\right\|_{\mathcal{L}^{\prime}}}{\varphi\left(2^{\ell} x, 2^{\ell} x\right)} \\
& \leq q d\left(f, T_{\ell} f\right)
\end{aligned}
$$


for all $x \in \mathcal{L}_{\overline{0}} \cup \mathcal{L}_{\overline{1}}$ and $\ell \in\{-1,1\}$. So,

$$
d\left(T_{\ell} f, T_{\ell}^{2} f\right) \leq q d\left(f, T_{\ell} f\right) .
$$

This means that $T$ is an orbitally continuous self-mapping of $\mathcal{E}$ satisfying (3). Since $\mathcal{L}^{\prime}$ is complete, then $(\mathcal{E}, d)$ is a generalized $T$-orbitally complete metric space.

Putting $y=x$ and dividing both sides of (7) by 2 , we get

$$
\left\|\frac{1}{2} f_{i}(2 x)-f_{i}(x)\right\|_{\mathcal{L}^{\prime}} \leq \varphi(x, x) / 2=q^{0} \varphi(x, x) / 2
$$

and then

$$
\left\|T_{1} f_{i}(x)-f_{i}(x)\right\|_{\mathcal{L}^{\prime}} \leq q^{0} \varphi(x, x) / 2
$$

for all $x \in \mathcal{L}_{\overline{0}} \cup \mathcal{L}_{\overline{1}}$ and $i \in \mathbb{Z}_{2}$.

Replacing $x$ and $y$ in (7) by $\frac{x}{2}$, we get

$$
\left\|f_{i}(x)-2 f_{i}\left(\frac{x}{2}\right)\right\|_{\mathcal{L}^{\prime}} \leq \varphi\left(\frac{x}{2}, \frac{x}{2}\right) \leq q^{1} \varphi(x, x) / 2
$$

and then

$$
\left\|f_{i}(x)-T_{-1} f_{i}(x)\right\|_{\mathcal{L}^{\prime}} \leq q^{1} \varphi(x, x) / 2
$$

for all $x \in \mathcal{L}_{\overline{0}} \cup \mathcal{L}_{\overline{1}}$ and $i \in \mathbb{Z}_{2}$.

It follows from (10) and (11) that, for $\ell \in\{-1,1\}$ and $i \in \mathbb{Z}_{2}$,

$$
d\left(f_{i}, T_{\ell} f_{i}\right) \leq q^{\frac{1-\ell}{2}} \varphi(x, x) / 2<\infty
$$

Now, it follows from Theorem 3.3 that there exists a fixed point $H_{i}^{\ell}$ of $T_{\ell}$ in $(\mathcal{E}, d)$ such that

$$
H_{i}^{\ell}(x)=\lim _{n \rightarrow \infty} T_{\ell}^{n} f_{i}(x)=\lim _{n \rightarrow \infty} 2^{-n \ell} f_{i}\left(2^{n \ell} x\right)
$$

and

$$
\left\|H_{i}^{\ell}(x)-f_{i}(x)\right\|_{\mathcal{L}^{\prime}} \leq \frac{q^{\frac{1-\ell}{2}}}{2(1-q)} \varphi(x, x)
$$

for all $x \in \mathcal{L}_{\overline{0}} \cup \mathcal{L}_{\overline{1}}, \ell \in\{-1,1\}$, and $i \in \mathbb{Z}_{2}$.

Let $x$ and $y$ be any two points of $\mathcal{L}_{\overline{0}} \cup \mathcal{L}_{\overline{1}}$. It follows from (7) that

$$
\begin{aligned}
& \left\|2^{-n \ell} f_{i}\left(2^{n \ell}(x+y)\right)-2^{-n \ell} f_{i}\left(2^{n \ell} x\right)-2^{-n \ell} f_{i}\left(2^{n \ell} y\right)\right\|_{\mathcal{L}^{\prime}} \\
& \quad \leq 2^{-n \ell} \varphi\left(2^{n \ell} x, 2^{n \ell} y\right) \leq q^{n} \varphi(x, y)
\end{aligned}
$$

and $n$ tending to infinity, we get

$$
H_{i}^{\ell}(x+y)=H_{i}^{\ell}(x)+H_{i}^{\ell}(y)
$$

for $\ell \in\{-1,1\}$ and $i \in \mathbb{Z}_{2}$. We see that for $\ell \in\{-1,1\}$ and $i \in \mathbb{Z}_{2}, H_{i}^{\ell}$ is additive. 
Claim that for each $\ell \in\{-1,1\}$ and $i \in \mathbb{Z}_{2}$, the function $H_{i}^{\ell}$ satisfies (1). It follows from (8) that

$$
\begin{aligned}
& \left\|H_{i}^{\ell}(\mu(x, y))-v\left(H_{i}^{\ell}(x), H_{i}^{\ell}(y)\right)\right\|_{\mathcal{L}^{\prime}} \\
& \quad=\lim _{n \rightarrow \infty} 2^{-2 n \ell}\left\|f_{i}\left(\mu\left(2^{n \ell} x, 2^{n \ell} y\right)\right)-v\left(f_{i}\left(2^{n \ell} x\right), f_{i}\left(2^{n \ell} y\right)\right)\right\|_{\mathcal{L}^{\prime}} \\
& \quad \leq \lim _{n \rightarrow \infty} 2^{-2 n \ell} \psi\left(2^{n \ell} x, 2^{n \ell} y\right) \\
& \leq \lim _{n \rightarrow \infty} q^{n} \psi(x, y)=0
\end{aligned}
$$

for all $x, y \in \mathcal{L}_{\overline{0}} \cup \mathcal{L}_{\overline{1}}, \ell \in\{-1,1\}$, and $i \in \mathbb{Z}_{2}$. So, we can conclude that

$$
H_{i}^{\ell}(\mu(x, y))=v\left(H_{i}^{\ell}(x), H_{i}^{\ell}(y)\right)
$$

for all $x, y \in \mathcal{L}_{\overline{0}} \cup \mathcal{L}_{\overline{1}}, \ell \in\{-1,1\}$, and $i \in \mathbb{Z}_{2}$. Now, it follows from Definition 2.1 that $H^{\ell}=$ $H_{\overline{0}}^{\ell}+H_{\overline{1}}^{\ell}$ is superhomomorphism and the proof is completed.

Example 4.2 Let $\mathcal{L}=\mathcal{L}_{\overline{0}} \oplus \mathcal{L}_{\overline{1}}$ be a Lie superalgebra with multiplication $\mu$ and norm $\|\cdot\|$. Consider the functions $f_{i}: \mathcal{L} \rightarrow \mathcal{L}\left(i \in \mathbb{Z}_{2}\right)$ defined by $f_{i}(x)=x+c$, where $0 \neq c \in \mathcal{L}^{c}$, for all $x \in \mathcal{L}_{\overline{0}} \cup \mathcal{L}_{\overline{1}}$ and $i \in \mathbb{Z}_{2}$. Define the functions $\varphi: \mathcal{L}^{2} \rightarrow(0, \infty)$ and $\psi: \mathcal{L}^{2} \rightarrow[0, \infty)$ by

$$
\varphi(x, y)=\psi(x, y)=\|c\| e^{\frac{1}{\|x\|}}
$$

for all $x, y \in \mathcal{L}_{\overline{0}} \cup \mathcal{L}_{\overline{1}}$ with $x \neq 0$. It is easy to see that $\varphi$ and $\psi$ satisfy the contractive conditions (5) and (6) for $q=q(1)=1 / 2$. For $x, y \in \mathcal{L}_{\overline{0}} \cup \mathcal{L}_{\overline{1}}$ with $x \neq 0$ and $i \in \mathbb{Z}_{2}$ we have

$$
\begin{aligned}
\left\|f_{i}(x+y)-f_{i}(x)-f_{i}(y)\right\| & =\|(x+y)+c-(x+c)-(y+c)\| \\
& =\|c\| \leq\|c\| e^{\frac{1}{\|x\|}}=\varphi(x, y)
\end{aligned}
$$

and

$$
\begin{aligned}
\left\|f_{i}(\mu(x, y))-\mu\left(f_{i}(x), f_{i}(y)\right)\right\| & =\|(\mu(x, y))+c-\mu(x+c, y+c)\| \\
& =\|(\mu(x, y))+c-\mu(x, y)-\mu(x, c)-\mu(c, y)-\mu(c, c)\| \\
& =\|c\| \leq\|c\| e^{\frac{1}{\|x\|}}=\psi(x, y) .
\end{aligned}
$$

Thus, by Theorem 4.1, there exists a unique superhomomorphism $H^{1}: \mathcal{L} \rightarrow \mathcal{L}^{\prime}$ with $H^{1}=$ $H_{\overline{0}}^{1}+H_{\overline{1}}^{1}$, where $H_{\overline{0}}^{1}$ and $H_{\overline{1}}^{1}$ are defined as

$$
\begin{aligned}
& H_{0}^{1}(x)=\lim _{n \rightarrow \infty} \frac{f_{0}\left(2^{n} x\right)}{2^{n}}=\lim _{n \rightarrow \infty} \frac{2^{n} x+c}{2^{n}}=x, \\
& H_{1}^{1}(x)=\lim _{n \rightarrow \infty} \frac{f_{1}\left(2^{n} x\right)}{2^{n}}=\lim _{n \rightarrow \infty} \frac{2^{n} x+c}{2^{n}}=x .
\end{aligned}
$$

Moreover, (9) is sharp. Indeed,

$$
\left\|H_{i}^{1}(x)-f_{i}(x)\right\|=\|x+c-x\|=\|c\| \leq \frac{(1 / 2)^{0}}{2(1-1 / 2)}\|c\| e^{\frac{1}{\|x\|}}=\|c\| e^{\frac{1}{\|x\|}}
$$

for all $x \in \mathcal{L}_{\overline{0}} \cup \mathcal{L}_{\overline{1}}$ with $x \neq 0$ and $i \in \mathbb{Z}_{2}$. 
In the next theorem, we prove the hyperstability of the superhomomorphism.

Theorem 4.3 Let $\mathcal{L}=\mathcal{L}_{\overline{0}} \oplus \mathcal{L}_{\overline{1}}$ be a Lie superalgebra with multiplication $\mu$ and norm $\|\cdot\|_{\mathcal{L}}$ and $\mathcal{L}^{\prime}=\mathcal{L}_{\overline{0}}^{\prime} \oplus \mathcal{L}_{\overline{1}}^{\prime}$ be a complete Lie superalgebra with multiplication $v$ and norm $\|\cdot\|_{\mathcal{L}^{\prime}}$. Assume that $f_{i}: \mathcal{L} \rightarrow \mathcal{L}^{\prime}\left(i \in \mathbb{Z}_{2}\right)$ are functions such that $f_{i}(2 x)=2 f_{i}(x)$ and

$$
\begin{aligned}
& \left\|f_{i}(x+y)-f_{i}(x)-f_{i}(y)\right\|_{\mathcal{L}^{\prime}} \leq \varphi(x, y), \\
& \left\|f_{i}(\mu(x, y))-v\left(f_{i}(x), f_{i}(y)\right)\right\|_{\mathcal{L}^{\prime}} \leq \psi(x, y)
\end{aligned}
$$

for all $x, y \in \mathcal{L}_{\overline{0}} \cup \mathcal{L}_{\overline{1}}$, where $\varphi: \mathcal{L}^{2} \rightarrow(0, \infty)$ and $\psi: \mathcal{L}^{2} \rightarrow[0, \infty)$ are functions satisfying the contractive conditions (5) and (6), for $0<q=q(\ell)<1$. Then $f=f_{\overline{0}}+f_{\overline{1}}$ is superhomomorphism.

Proof If we replace $x$ and $y$ in (12) with $2^{n} x$ and $2^{n} y$, respectively, and divide by $2^{n}$ the resulting inequality, then we have

$$
\left\|f_{i}(x+y)-f_{i}(x)-f_{i}(y)\right\|_{\mathcal{L}^{\prime}} \leq 2^{-n} \varphi\left(2^{n} x, 2^{n} y\right) \leq q^{n} \varphi(x, y)
$$

for all $x, y \in \mathcal{L}_{\overline{0}} \cup \mathcal{L}_{\overline{1}}, i \in \mathbb{Z}_{2}$, and $n \in \mathbb{N}$.

If we replace $x$ and $y$ in (13) with $2^{n} x$ and $2^{n} y$, respectively, and divide by $2^{2 n}$ the resulting inequality, then we have

$$
\left\|f_{i}(\mu(x, y))-v\left(f_{i}(x), f_{i}(y)\right)\right\|_{\mathcal{L}^{\prime}} \leq 2^{-2 n} \psi\left(2^{n} x, 2^{n} y\right) \leq q^{n} \psi(x, y)
$$

for all $x, y \in \mathcal{L}_{\overline{0}} \cup \mathcal{L}_{\overline{1}}, i \in \mathbb{Z}_{2}$ and $n \in \mathbb{N}$. From $n \rightarrow \infty$ in (14) and (15), we conclude that $f_{\overline{0}}$ and $f_{\overline{1}}$ are homomorphisms and hence $f=f_{\overline{0}}+f_{\overline{1}}$ is superhomomorphism.

\section{The superderivation in Lie superalgebras}

Theorem 5.1 Let $\mathcal{L}=\mathcal{L}_{\overline{0}} \oplus \mathcal{L}_{\overline{1}}$ be a complete Lie superalgebra with multiplication $\mu$ and norm $\|\cdot\|_{\mathcal{L}}$. Let $f_{i}: \mathcal{L} \rightarrow \mathcal{L}\left(i \in \mathbb{Z}_{2}\right)$ be functions satisfying

$$
\begin{aligned}
& \left\|f_{i}(x+y)-f_{i}(x)-f_{i}(y)\right\|_{\mathcal{L}} \leq \varphi(x, y), \\
& \left\|f_{i}(\mu(x, y))-\mu\left(f_{i}(x), y\right)-(-1)^{i|x|} \mu\left(x, f_{i}(y)\right)\right\|_{\mathcal{L}} \leq \psi(x, y)
\end{aligned}
$$

for all $x, y \in \mathcal{L}_{\overline{0}} \cup \mathcal{L}_{\overline{1}}$, where $\varphi: \mathcal{L}^{2} \rightarrow(0, \infty)$ and $\psi: \mathcal{L}^{2} \rightarrow[0, \infty)$ are functions satisfying the contractive conditions (5) and (6), for $0<q=q(\ell)<1$. Then there exists a unique superderivation $D^{\ell}: \mathcal{L} \rightarrow \mathcal{L}$ with $D^{\ell}=D_{\overline{0}}^{\ell}+D_{\overline{1}}^{\ell}$ such that

$$
\left\|D_{i}^{\ell}(x)-f_{i}(x)\right\|_{\mathcal{L}} \leq \frac{q^{\frac{1-\ell}{2}}}{2(1-q)} \varphi(x, x)
$$

for all $x \in \mathcal{L}_{\overline{0}} \cup \mathcal{L}_{\overline{1}}, \ell \in\{-1,1\}$, and $i \in \mathbb{Z}_{2}$.

Proof Since the functions $f_{i}\left(i \in \mathbb{Z}_{2}\right)$ satisfy (16), according to the corresponding part of Theorem 4.1, we can conclude that there exists a unique additive function $D^{\ell}: \mathcal{L} \rightarrow \mathcal{L}$ 
with $D^{\ell}=D_{\overline{0}}^{\ell}+D_{\overline{1}}^{\ell}$ and defined by

$$
D_{i}^{\ell}(x)=\lim _{n \rightarrow \infty} 2^{-n \ell} f_{i}\left(2^{n \ell} x\right)
$$

such that

$$
\left\|D_{i}^{\ell}(x)-f_{i}(x)\right\|_{\mathcal{L}} \leq \frac{q^{\frac{1-\ell}{2}}}{2(1-q)} \varphi(x, x)
$$

for all $x \in \mathcal{L}_{\overline{0}} \cup \mathcal{L}_{\overline{1}}, \ell \in\{-1,1\}$, and $i \in \mathbb{Z}_{2}$. We only need to show that $D_{\overline{0}}^{\ell}$ and $D_{\overline{1}}^{\ell}$ satisfy (2). It follows from (17) that

$$
\begin{aligned}
& \left\|D_{i}^{\ell}(\mu(x, y))-\mu\left(D_{i}^{\ell}(x), y\right)-(-1)^{i|x|} \mu\left(x, D_{i}^{\ell}(y)\right)\right\|_{\mathcal{L}} \\
& \quad=\lim _{n \rightarrow \infty} 2^{-2 n \ell}\left\|f_{i}\left(\mu\left(2^{n \ell} x, 2^{n \ell} y\right)\right)-\mu\left(f_{i}\left(2^{n \ell} x\right), 2^{n \ell} y\right)-(-1)^{i|x|} \mu\left(2^{n \ell} x, f_{i}\left(2^{n \ell} y\right)\right)\right\|_{\mathcal{L}} \\
& \quad \leq \lim _{n \rightarrow \infty} 2^{-2 n \ell} \psi\left(2^{n \ell} x, 2^{n \ell} y\right) \\
& \quad \leq \lim _{n \rightarrow \infty} q^{n} \psi(x, y)=0
\end{aligned}
$$

for all $x, y \in \mathcal{L}_{\overline{0}} \cup \mathcal{L}_{\overline{1}}, \ell \in\{-1,1\}$, and $i \in \mathbb{Z}_{2}$. It is easy to see that

$$
D_{i}^{\ell}(\mu(x, y))=\mu\left(D_{i}^{\ell}(x), y\right)+(-1)^{i|x|} \mu\left(x, D_{i}^{\ell}(y)\right)
$$

for all $x, y \in \mathcal{L}_{\overline{0}} \cup \mathcal{L}_{\overline{1}}, \ell \in\{-1,1\}$, and $i \in \mathbb{Z}_{2}$. It follows from Definition 2.2 that $D^{\ell}=D_{\overline{0}}^{\ell}+D_{\overline{1}}^{\ell}$ is superderivation and the proof is completed.

In the next theorem, we present the hyperstability result concerning the superderivation in Lie superalgebras.

Theorem 5.2 Let $\mathcal{L}=\mathcal{L}_{\overline{0}} \oplus \mathcal{L}_{\overline{1}}$ be a complete Lie superalgebra with multiplication $\mu$ and norm $\|\cdot\|_{\mathcal{L}}$. Let $f_{i}: \mathcal{L} \rightarrow \mathcal{L}\left(i \in \mathbb{Z}_{2}\right)$ be functions such that $f_{i}(2 x)=2 f_{i}(x)$ and

$$
\begin{aligned}
& \left\|f_{i}(x+y)-f_{i}(x)-f_{i}(y)\right\|_{\mathcal{L}} \leq \varphi(x, y), \\
& \left\|f_{i}(\mu(x, y))-\mu\left(f_{i}(x), y\right)-(-1)^{i|x|} \mu\left(x, f_{i}(y)\right)\right\|_{\mathcal{L}} \leq \psi(x, y)
\end{aligned}
$$

for all $x, y \in \mathcal{L}_{\overline{0}} \cup \mathcal{L}_{\overline{1}}$, where $\varphi: \mathcal{L}^{2} \rightarrow(0, \infty)$ and $\psi: \mathcal{L}^{2} \rightarrow[0, \infty)$ are functions satisfying the contractive conditions (5) and (6), for $0<q=q(\ell)<1$. Then $f=f_{\overline{0}}+f_{\overline{1}}$ is superderivation.

Proof Replacing $x$ and $y$ in (18) with $2^{n} x$ and $2^{n} y$, respectively, and dividing by $2^{n}$ the resulting inequality, we get

$$
\left\|f_{i}(x+y)-f_{i}(x)-f_{i}(y)\right\|_{\mathcal{L}} \leq 2^{-n} \varphi\left(2^{n} x, 2^{n} y, 0\right) \leq q^{n} \varphi(x, y)
$$

for all $x, y \in \mathcal{L}_{\overline{0}} \cup \mathcal{L}_{\overline{1}}, i \in \mathbb{Z}_{2}$ and $n \in \mathbb{N}$.

Replacing $x$ and $y$ in (19) with $2^{n} x$ and $2^{n} y$, respectively, and dividing by $2^{2 n}$ the resulting inequality, we obtain

$$
\left\|f_{i}(\mu(x, y))-\mu\left(f_{i}(x), y\right)-(-1)^{i|x|} \mu\left(x, f_{i}(y)\right)\right\|_{\mathcal{L}} \leq 2^{-2 n} \psi\left(2^{n} x, 2^{n} y\right) \leq q^{n} \psi(x, y)
$$


for all $x, y \in \mathcal{L}_{\overline{0}} \cup \mathcal{L}_{\overline{1}}, i \in \mathbb{Z}_{2}$, and $n \in \mathbb{N}$. From $n \rightarrow \infty$ in (20) and (21), we conclude that $f_{\overline{0}}$ and $f_{\overline{1}}$ are derivations and $f=f_{\overline{0}}+f_{\overline{1}}$ is a superderivation.

\section{An application of Theorem 3.3}

In 1978, Rassias proved the following theorem concerning the stability of functional equations.

Theorem 6.1 (Rassias [11]) Consider $E_{1}, E_{2}$ to be two Banach spaces, and let $f: E_{1} \rightarrow E_{2}$ be a mapping such that $f(t x)$ is continuous in $t$ for each fixed $x$. Assume that there exist $\theta \geq 0$ and $0 \leq p<1$ such that

$$
\frac{\|f(x+y)-f(x)-f(y)\|_{E_{2}}}{\|x\|_{E_{1}}^{p}+\|y\|_{E_{1}}^{p}} \leq \theta \quad \text { for any } x, y \in E_{1} .
$$

Then there exists a unique linear mapping $A: E_{1} \rightarrow E_{2}$ such that

$$
\frac{\|f(x)-A(x)\|_{E_{2}}}{\|x\|_{E_{1}}^{p}} \leq \frac{2 \theta}{2-2^{p}} \quad \text { for any } x \in E_{1} .
$$

In this section, we show that Theorem 6.1, concerning the stability of the Cauchy functional equation in Banach spaces, is a direct consequence of Theorem 3.3. In general, almost all of the theorems concerning the stability problem of different functional equations are consequences of Theorem 3.3.

Proof Let us consider the set $X:=\left\{g: E_{1} \rightarrow E_{2}: g(0)=0\right\}$ and introduce a generalized metric on $X$ :

$$
d(g, h)=\sup _{x \neq 0} \frac{\|g(x)-h(x)\|_{E_{2}}}{\|x\|_{E_{1}}^{p}} .
$$

Consider the linear mapping $T: X \rightarrow X$ defined as

$$
\operatorname{Tg}(x)=2^{-1} g(2 x)
$$

for all $x \in E_{1}$. We can write, for any $f \in X$,

$$
\begin{aligned}
\frac{\left\|T f(x)-T^{2} f(x)\right\|_{E_{2}}}{\|x\|_{E_{1}}^{p}} & =\frac{\left\|2^{-1} f(2 x)-4^{-1} f(4 x)\right\|_{E_{2}}}{\|x\|_{E_{1}}^{p}} \\
& =2^{p-1} \frac{\left\|f(2 x)-2^{-1} f(4 x)\right\|_{E_{2}}}{\|2 x\|_{E_{1}}^{p}} \\
& \leq 2^{p-1} d(f, T f)
\end{aligned}
$$

for all $x \in E_{1}$. So,

$$
d\left(T f, T^{2} f\right) \leq 2^{p-1} d(f, T f) \quad(0 \leq p<1)
$$

This means that $T$ is an orbitally continuous self-mapping of $X$ satisfying (3) with $q=2^{p-1}$. Since $E_{2}$ is complete, then $(X, d)$ is a generalized $T$-orbitally complete metric space. 
By equation (22), one can easily show that

$$
\frac{\|f(x)-f(2 x) / 2\|}{\|x\|^{p}} \leq \theta<\infty .
$$

Now, it follows from Theorem 3.3 that there exists a unique fixed point $A$ of $T$ in $(X, d)$ such that

$$
A(x)=\lim _{n \rightarrow \infty} T^{n} f(x)=\lim _{n \rightarrow \infty} 2^{-n} f\left(2^{n} x\right)
$$

and

$$
\frac{\|f(x)-A(x)\|_{E_{2}}}{\|x\|_{E_{1}}^{p}} \leq \frac{1}{1-2^{p-1}} d(f, T f)=\frac{1}{1-2^{p-1}} \theta
$$

for all $x \in E_{1}$. It is clear that $A$ is linear and the proof is completed.

Competing interests

The authors declare that they have no competing interests.

\section{Authors' contributions}

The authors confirm that the manuscript has been read and approved by all named authors and that there are no other persons who satisfied the criteria for authorship but are not listed. The authors further confirm that the order of authors listed in the manuscript has been approved by all of them. All authors contributed equally to the writing of this paper. All authors read and approved the final manuscript.

\section{Author details}

1 Department of Mathematics, Faculty of Mathematics, Statistics and Computer Sciences, Semnan University, Semnan, 35195-363, Iran. ${ }^{2}$ Center of Excellence in Nonlinear Analysis and Applications, Semnan University, Semnan, Iran. ${ }^{3}$ Young Researchers and Elite Club, Malayer Branch, Azad University, Malayer, Iran. ${ }^{4}$ Department of Electricity and Electronics, University of the Basque Country, 466, Bilbao, 48080, Spain.

\section{Acknowledgements}

The authors are very grateful to the Spanish Government for its support of this research through Grant DPI2012-30651. The authors are also grateful to the Basque Government through Grant IT 378-10.

Received: 23 April 2015 Accepted: 16 November 2015 Published online: 26 November 2015

\section{References}

1. Abellanas, L, Alonso, L: A general setting for Casimir invariants. J. Math. Phys. 16, 1580-1584 (1975)

2. Jacobson, N: Lie Algebras. Dover, New York (1979)

3. Rand, D, Winternitz, P, Zassenhaus, H: On the identification of Lie algebra given by its structure constants I. Direct decompositions, Levi decompositions and nilradicals. Linear Algebra Appl. 109, 197-246 (1988)

4. Popovych, R, Boyko, V, Nesterenko, M, Lutfullin, M: Realizations of real low-dimensional Lie algebras. J. Phys. A 36, 7337-7360 (2003)

5. Montigny, M, Patera, J: Discrete and continuous graded contractions of Lie algebras and superalgebras. J. Algebra 24, 525-547 (1991)

6. Lada, T, Stasheff, J: Introduction to SH Lie algebras for physicists. Int. J. Theor. Phys. 32, 1087-1103 (1993)

7. de Azcárraga, JA, Bueno, JCP: Higher-order simple Lie algebras. Commun. Math. Phys. 184, 669-681 (1997)

8. Palev, TD: Lie superalgebras, infinite-dimensional algebras and quantum statistics. Rep. Math. Phys. 31, 241-262 (1992)

9. Ulam, SM: A Collection of Mathematical Problems. Interscience, New York (1960)

10. Hyers, DH: On the stability of the linear functional equation. Proc. Natl. Acad. Sci. USA 27, $222-224$ (1941)

11. Rassias, TM: On the stability of the linear mapping in Banach spaces. Proc. Am. Math. Soc. 72, 297-300 (1978)

12. Baker, JA: The stability of certain functional equations. Proc. Am. Math. Soc. 112, 729-732 (1991)

13. Baker, JA, Lawrence, J, Zorzitto, F: The stability of the equation $f(x+y)=f(x) f(y)$. Proc. Am. Math. Soc. 74, 242-246 (1979)

14. Rassias, TM: On the stability of functional equations in Banach spaces. J. Math. Anal. Appl. 251, 264-284 (2000)

15. Šemrl, P: The functional equation of multiplicative derivation is superstable on standard operator algebras. Integral Equ. Oper. Theory 18, 118-122 (1994)

16. Gordji, ME: Nearly involutions on Banach algebras: a fixed point approach. J. Math. Phys. 14, 117-124 (2013)

17. Montgomery, S: Constructing simple Lie superalgebras from associative graded algebras. J. Algebra 195, 558-579 (1997) 
18. Fošner, M: On the extended centroid of prime associative superalgebras with applications to superderivations. Commun. Algebra 32, 689-705 (2004)

19. Chen, TS: Supercentralizing superderivations on prime superalgebras. Commun. Algebra 33, 4457-4466 (2005)

20. Zusmanovich, P: On $\delta$-derivations of Lie algebras and superalgebras. J. Algebra 324, 3470-3486 (2010)

21. Banach, S: Sur les operations dans les ensembles abstraites et leurs applications. Fundam. Math. 3, 133-181 (1922)

22. Agarwal, RP, Balaj, M, O'Regan, D: A common fixed point theorem with applications. J. Optim. Theory Appl. 163, 482-490 (2014)

23. Cho, YJ, Saadati, R, Wang, S: Common fixed point theorems on generalized distance in ordered cone metric spaces. Comput. Math. Appl. 61, 1254-1260 (2011)

24. Pathak, HK, Agarwal, RP, Cho, YJ: Coincidence and fixed points for multi-valued mappings and its application to nonconvex integral inclusions. J. Comput. Appl. Math. 283, 201-217 (2015)

25. Golkarmanesh, F, Al-Mazrooei, AE, Parvaneh, V, Latif, A: Fixed point results for generalized mappings. Fixed Point Theory Appl. 2014, 217 (2014)

26. Radu, $\mathrm{V}$ : The fixed point alternative and the stability of functional equations. Fixed Point Theory 4, 91-96 (2003)

27. Gordji, ME, Najati, A: Approximately J*-homomorphisms: a fixed point approach. J. Geom. Phys. 60, 809-814 (2010)

28. Chauhan, S, Pant, BD: Fixed point theorems for compatible and subsequentially continuous mappings in Menger spaces. J. Nonlinear Sci. Appl. 7, 78-89 (2014)

29. Miheț, D: Common coupled fixed point theorems for contractive mappings in fuzzy metric spaces. J. Nonlinear Sci. Appl. 6, 35-40 (2013)

30. Cirić, LB: Fixed and periodic points of almost contractive operators. Math. Balk. 3, 33-44 (1973)

31. Ćirić, LB: Fixed points for generalized multi-valued contractions. Mat. Vesn. 9, 265-272 (1972)

32. Ćirić, LB: On contraction type mappings. Math. Balk. 1, $52-57$ (1971)

33. Eshaghi, M, Abbaszadeh, S: Approximate generalized derivations close to derivations in Lie $C^{*}$-algebras. J. Appl. Anal. 21, 37-43 (2015)

34. Eshaghi, M, Abbaszadeh, S, de la Sen, M: On the stability of conditional homomorphisms in Lie $C^{*}$-algebras. J. Gen. Lie Theory Appl. 9, 220 (2015)

\section{Submit your manuscript to a SpringerOpen ${ }^{\circ}$ journal and benefit from:}

$\rightarrow$ Convenient online submission

Rigorous peer review

- Immediate publication on acceptance

- Open access: articles freely available online

- High visibility within the field

- Retaining the copyright to your article 